\title{
Effect of Twist on Transverse Impact Response of Ballistic Fiber Yarns
}

\author{
Bo Song ${ }^{1, a}$, Wei-Yang $\mathrm{Lu}^{2}$ \\ ${ }^{1}$ Sandia National Laboratories, Albuquerque, NM 87185; email: bsong@ sandia.gov \\ ${ }^{2}$ Sandia National Laboratories, Livermore, CA 94550
}

\begin{abstract}
A Hopkinson bar was employed to conduct transverse impact testing of twisted Kevlar KM2 fiber yarns at the same impact speed. The speed of Euler transverse wave generated by the impact was measured utilizing a high speed digital camera. The study included fiber yarns twisted by different amounts. The Euler transverse wave speed was observed to increase with increasing amount of twist of the fiber yarn, within the range of this investigation. The higher transverse wave speeds in the more twisted fiber yarns indicate better ballistic performance in soft body armors for personal protection.
\end{abstract}

Keywords: Transverse impact, ballistic fiber, twist, ballistic performance, Hopkinson bar 


\section{Introduction}

Effective and efficient soft body armor employed against ballistic or blast impact depends on the mechanical properties of the fiber yarns used in the fabric, and on the fabrication process. Ballistic performance of fiber yarns and fabrics has been investigated through theoretical analysis, numerical simulation, and experiments [1-4]. When a fiber yarn or fabric is subjected to external impact, a transverse wave is produced and propagates in the yarn or fabric. The speed of transverse wave has been found to be critical to their ballistic performance [5]. A faster transverse wave speed is desired to dissipate the impact energy quickly [5]. The transverse wave speed has been shown to depend on the external impact speed and the longitudinal wave speed that is usually a material constant of the fiber yarn [6].

When an aligned fiber yarn is subjected to transverse impact, the individual fibers in the yarn are impacted progressively along the thickness direction within the first several microseconds [6]. Figure 1 shows a high-speed image at the very early stage of a Kevlar ${ }^{\circledR}$ KM2 fiber yarn being impacted. As shown in Fig. 1, only the first half of fibers in the yarn were impacted and deformed at this moment while the other half of fibers kept staying free. This image reveals that not all fibers have been impacted simultaneously, which may result in progressive premature failure of the fiber yarn when subjected to high speed impact. In this case, the fiber yarn behaves simply as a group of individual fibers instead of a solid component bundling all individual fibers, which possibly downgrades the global ballistic performance. It is therefore reasonable to characterize the transverse impact response of twisted fiber yarns. In addition, the angle shown in Fig. 1 might represent the transverse wave propagation in the portion of the compressed fibers instead of the whole fiber yarn. The impact wedge was shaped but might still affect the shape of the deformed fibers at very early stage of impact loading. 
Twisting a fiber yarn has been explored to address the deficit in the mechanical response discussed above. Twist more effectively holds all individual fibers in a fiber yarn without significant change in density. For example, twist can improve the interactions among individual fibers in terms of friction forces such that the fibers are capable of bearing load even after some individual fibers are broken [7]. The mechanical properties of twisted fiber yarns have been investigated in [8-10]. Both modulus and tensile strength change after a fiber yarn is twisted. In general, the tensile strength increases when the fiber yarn is slightly twisted. However, the change in modulus has been observed to depend on the amount of twist. For example, Im et al. [10] found the tensile strength increased after the poly (paraphenylene benzobisoxazole) or PBO fiber yarn was twisted. The tensile strength kept increasing with increased twist level up to 6 turns per inch. When the fiber yarn was twisted more, the tensile strength decreased. The Young's modulus had little change when the fiber yarn was twisted by 6 twists per inch or under. However, when the fiber yarn was twisted more, the Young's modulus significantly decreased. Therefore, the twist level is a critical parameter that affects the mechanical properties of the fiber yarn.

There has been little to document the twist effect on the transverse wave speed produced in a fiber yarn subjected to transverse impact. In this paper, we report the transverse wave speed produced in twisted fiber yarns by different amount of twist to determine the effect of twist on the transverse wave speed and thus the ballistic performance.

\section{Experiment and Results}


The experimental procedure of the transverse impact on a fiber yarn has been documented in Ref. [6]. The schematic of this modified Hopkinson bar setup is shown in Fig. 2. In this study, the fiber yarn used was 450 denier Kevlar KM2 taken from a 0/90 plain woven fabric, which is the same as the fiber yarn in Ref. [6]. As shown in Fig. 2, the fiber yarn was set perpendicular to the pressure bar axis. The initial load on the fiber yarn was set to be nearly zero $(<0.3 \mathrm{~N})$ measured with a load cell attached to the bottom. A Cordin 550 high speed digital camera was used to take the images of the fiber deformation during transverse impact. In this study, we performed transverse impact on the fiber yarns at the same speed, $\mathrm{V}=53 \mathrm{~m} / \mathrm{s}$. The fiber yarns were twisted by different amounts: 0 (no twist), 1/3, 2/3, 4/3, and 8/3 turns per inch, before transverse impact.

The transverse impact experimental results show that all twisted fiber yarns displayed a very similar characteristic of deformation: an isosceles triangle shape was formed. With increasing time (or the bar end displacement), the triangle area became larger and both sides moved outwards along the non-deformed fiber yarn [6]. The movement of the sides along the fiber yarn represents the propagation of Euler transverse wave. Since the impact velocity of the pressure bar end was constant while deflecting the fiber yarn, there was little change in the internal angle of the isosceles triangle. There is a relationship between the angle, $\gamma$, marked in Fig. 3, and the Euler transverse wave speed, $c_{s}$, [6]

$c_{S}=\frac{V_{0}}{\tan \gamma}$

where $V_{0}$ is the impact velocity of the bar end. It is noted that, the velocity of the bar end is the same as the striker speed in this study where the resistance of the fiber yarn to the bar-end 
movement is negligible. We measured the angle for each test of the fiber yarns with different amount of twist and found that the angle was dependent on the twist amount. In other words, the twist amount influenced the transverse wave speed according to Eq. (1). The high-speed images for the fiber yarns, without twist and with a twist of $4 / 3$ turns per inch, were overlapped to compare the difference in angle upon impact at the same speed. The overlapped image is shown in Fig. 3. The blurry white lines in Fig. 3 are the fiber yarns which are also represented with the black lines to give a clearer comparison. At the same time (or the same bar end displacement), the twisted fiber yarn had a smaller angle than the non-twisted (or aligned) fiber yarn. The jump starting point in the twisted fiber yarn is ahead of that in the non-twisted (or aligned) fiber yarn, indicating a faster Euler transverse wave speed in the twisted fiber yarn.

We quantitatively measured the internal angles generated in the non-twisted and twisted fiber yarns, respectively, and calculated the corresponding Euler transverse wave speeds with Eq. (1). The results are listed in Table 1. Table 1 also lists the measured angles and calculated Euler transverse wave speed (Eq. (1)) in the fiber yarns with different amounts of twist. The Euler transverse wave speeds were also normalized by that in the non-twisted (or aligned) fiber yarn. The normalized Euler transverse wave speed data in Table 1 are also plotted in Fig. 4 to show the effect of twist amount on the Euler transverse wave speed. Figure 4 clearly shows that, at the same impact speed, the Euler transverse wave speed nonlinearly increases with increasing twist amount in the fiber yarn. For example, when the fiber yarn was twisted by $1 / 3$ turns per inch, the Euler transverse wave speed was increased by $6 \%$. The Euler transverse wave speed was even increased by over $20 \%$ in the fiber yarn twisted by $8 / 3$ turns per inch. Figure 4 also shows that the rate of change in Euler transverse wave speed decreases with increasing twist amount. The 
Euler transverse wave speed may decrease due to possible reduction in Young's modulus with increasing twist amount [8-10].

It was concluded in [6] that the longitudinal wave speed, $C_{l}$, in a linear elastic fiber yarn is a key parameter that affects the Euler transverse wave speed at a certain impact speed, $V_{0}$, through the equation

$V_{0}=\frac{c_{s}}{C_{l}-2 c_{s}} \sqrt{c_{s}\left(2 C_{l}-3 c_{s}\right)}$

In the case of $c_{s} \ll C_{l}$, we have

$c_{S}=\left(\frac{C_{l}}{2}\right)^{1 / 3} V_{0}^{2 / 3}$

Both Eqs. (2) and (3) indicate a fiber yarn with a higher longitudinal wave speed produces a higher Euler transverse wave speed at a certain impact speed. It turns out that the longitudinal wave speed may increase when the fiber yarn is twisted such that the Euler transverse wave speed increases. For a linear elastic material, the longitudinal wave speed depends on Young's modulus, $E$, and density of the fiber yarn, $\rho$,

$C_{l}=\sqrt{\frac{E}{\rho}}$

There is little difference in density between twisted and non-twisted fiber yarns. Therefore, the Young's moduli increase in the twisted Kevlar fiber yarns. However, there is no such data in 
regard to either Young's modulus or longitudinal wave speed available for the twisted Kevlar KM2 fiber yarns. Experimental techniques are motivated to be developed to directly measure the longitudinal wave speed in twisted fiber yarns. In this short communication, we are reporting the effect of twist amount on the Euler transverse wave speed only. The experimental techniques for measuring longitudinal wave speeds in fiber yarns may be discussed in future work.

\section{Conclusions}

We employed a Hopkinson bar apparatus to characterize the transverse impact response of Kevlar KM2 fiber yarns with different amounts of twist. The fiber yarns were twisted by 0 (non-twisted), 1/3,2/3,4/3, and $8 / 3$ turns per inch, respectively, and then subjected to a constant transverse impact speed of $53 \mathrm{~m} / \mathrm{s}$. All fiber yarns exhibited very similar transverse impact response. A transverse wave was formed and propagated outwards along the fiber yarn. However, the speed of Euler transverse wave depended on the amount of twist. The Euler transverse wave propagated at higher speeds in the twisted fiber yarns. Within the twist range in this study, the Euler transverse wave speed increased with increasing twist amount. The ballistic performance can therefore be improved by using appropriately twisted fiber yarns in a ballistic fabric.

\section{Acknowledgements}

Sandia National Laboratories is a multi-program laboratory managed and operated by Sandia Corporation, a wholly owned subsidiary of Lockheed Martin Corporation, for the U.S. Department of Energy's National Nuclear Security Administration under contract DE-AC0494AL85000. 


\section{References}

[1] Smith, J. C., McCrackin, F. L., Schiefer, H. F., Stone, W. K., and Towne, K. M., 1956, "Stress-Strain Relationships in Yarns Subjected to Rapid Impact Loading: Part IV: Transverse Impact Tests,” Textile Research Journal, 26, 821-828.

[2] Smith, J. C., McCrackin, F. L., and Schiefer, H. F., 1958, "Stress-Strain Relationships in Yarns Subjected to Rapid Impact Loading: Part V: Wave Propagation in Long Textile Yarns Impacted Transversely," Textile Research Journal, 28, 288-302.

[3] Cunniff, P. M., 1992, “An Analysis of the System Effects of Woven Fabrics under Ballistic Impact," Textile Research Journal, 62, 495-509.

[4] Chocron, S., Kirchdoerfer, T., King, N., and Freitas, C. J., 2011, "Modeling of Fabric Impact with High Speed Imaging and Nickel-Chromium Wires Validation,” Transactions of the ASME, Journal of Applied Mechanics, 78, 051007-1-13.

[5] Wang, L., 2007, Foundations of Stress Waves, Elsevier, Oxford.

[6] Song, B., Park, H., Lu, W.-Y., and Chen, W., 2011, Transverse Impact Response of a Linear Elastic Ballistic Fiber Yarn, Transactions of the ASME, Journal of Applied Mechanics, 78, 051023-1-9.

[7] Porwal, P. K., Beyerlein, I. J., and Phoenix, S. L., 2007, Statistical Strength of Twisted Fiber Bundles with Load Sharing Controlled by Frictional Length Scales, Journal of Mechanics of Materials and Structures, 2, 773-791. 
[8] Rao, Y., and Farris, R. J., 2000, A Modeling and Experimental Study of the Influence of Twist on the Mechanical Properties of High-Performance Fiber Yarns, Journal of Applied Polymer Science, 77, 1938-1949.

[9] Weinberg, A., and Schwartz, P., 1987, Twist Effects on the Mechanical Behaviour of Kevlar 29/Epoxy Strands, Journal of Materials Science Letters, 6, 832-834.

[10] Im, J., Percha, P. A., and Yeakle, D. S., 1989, "Some Physical and Mechanical Properties of PBO Fiber,” Mat. Res. Soc. Symp. Proc., 134, 307-312. 
Table 1. Twist Effect on Euler Transverse Wave Speed at the Same Impact Speed of $53 \mathrm{~m} / \mathrm{s}$

\begin{tabular}{|c|c|c|c|}
\hline $\begin{array}{c}\text { Twist Amount } \\
\text { (twist per inch) }\end{array}$ & Angle & $\begin{array}{c}\text { Euler Transverse } \\
\text { Wave Speed } \\
c_{S}(\mathrm{~m} / \mathrm{s})\end{array}$ & $\begin{array}{c}\text { Normalized Euler } \\
\text { Transverse Wave } \\
\text { Speed, } c_{s} / c_{s 0}\end{array}$ \\
\hline 0 & $19.7^{\circ}$ & 148.0 & 1 \\
\hline $1 / 3$ & $18.6^{\circ}$ & 157.5 & 1.064 \\
\hline $2 / 3$ & $17.6^{\circ}$ & 167.1 & 1.129 \\
\hline $4 / 3$ & $17.1^{\circ}$ & 172.3 & 1.164 \\
\hline $8 / 3$ & $16.5^{\circ}$ & 178.9 & 1.209 \\
\hline
\end{tabular}




\section{List of Figure Captions}

Figure 1. Non-uniformly transverse impact on the non-twisted fiber yarn

Figure 2. Schematic of the Hopkinson bar for transverse impact testing of fiber yarns

Figure 3. Comparison of Euler transverse wave propagation in non-twisted and twisted fiber yarns

Figure 4. Effect of twist amount on Euler transverse wave speed at the same impact speed of 53 $\mathrm{m} / \mathrm{s}$. 


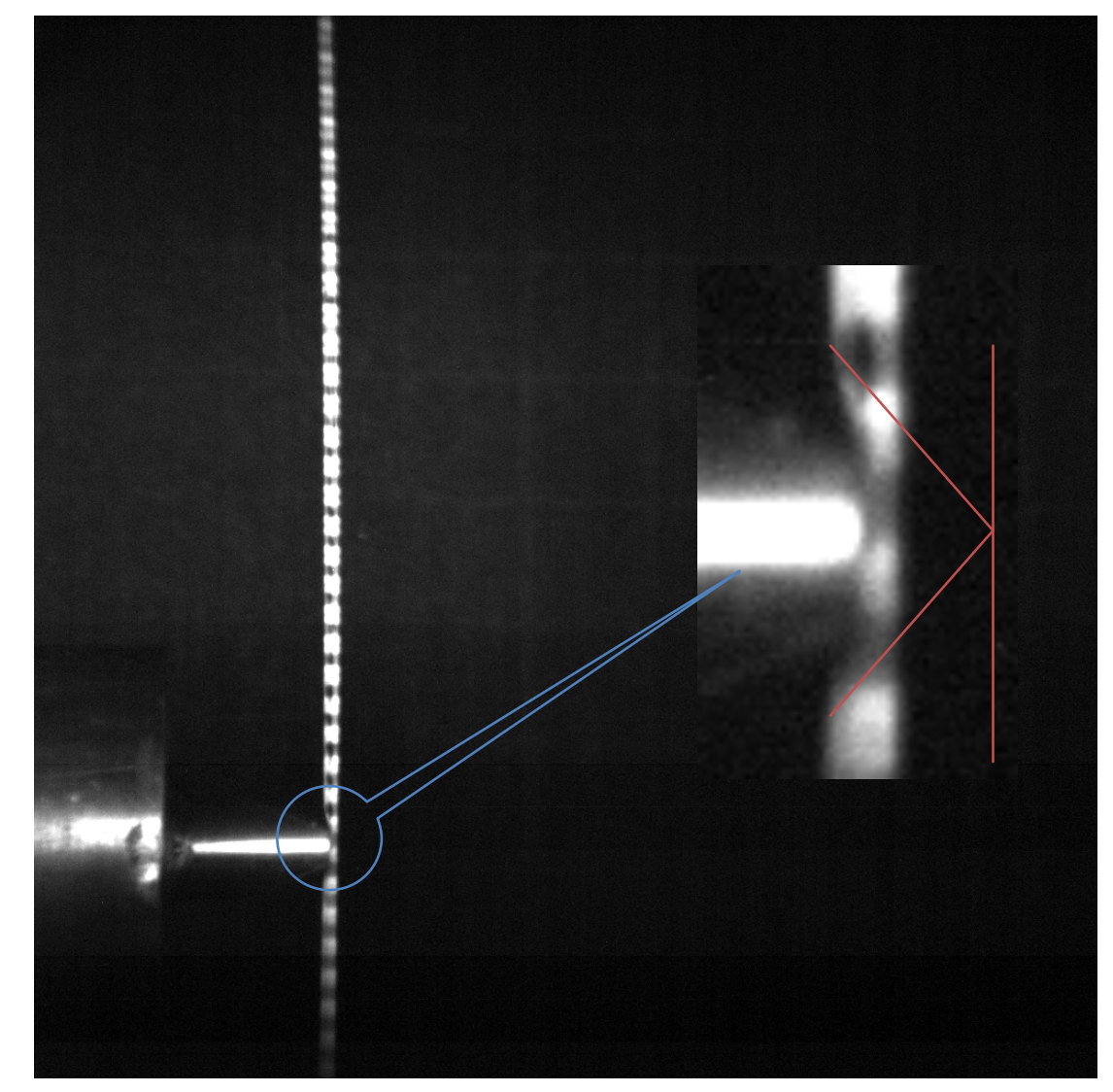

Figure 1

Figures

Figure 1

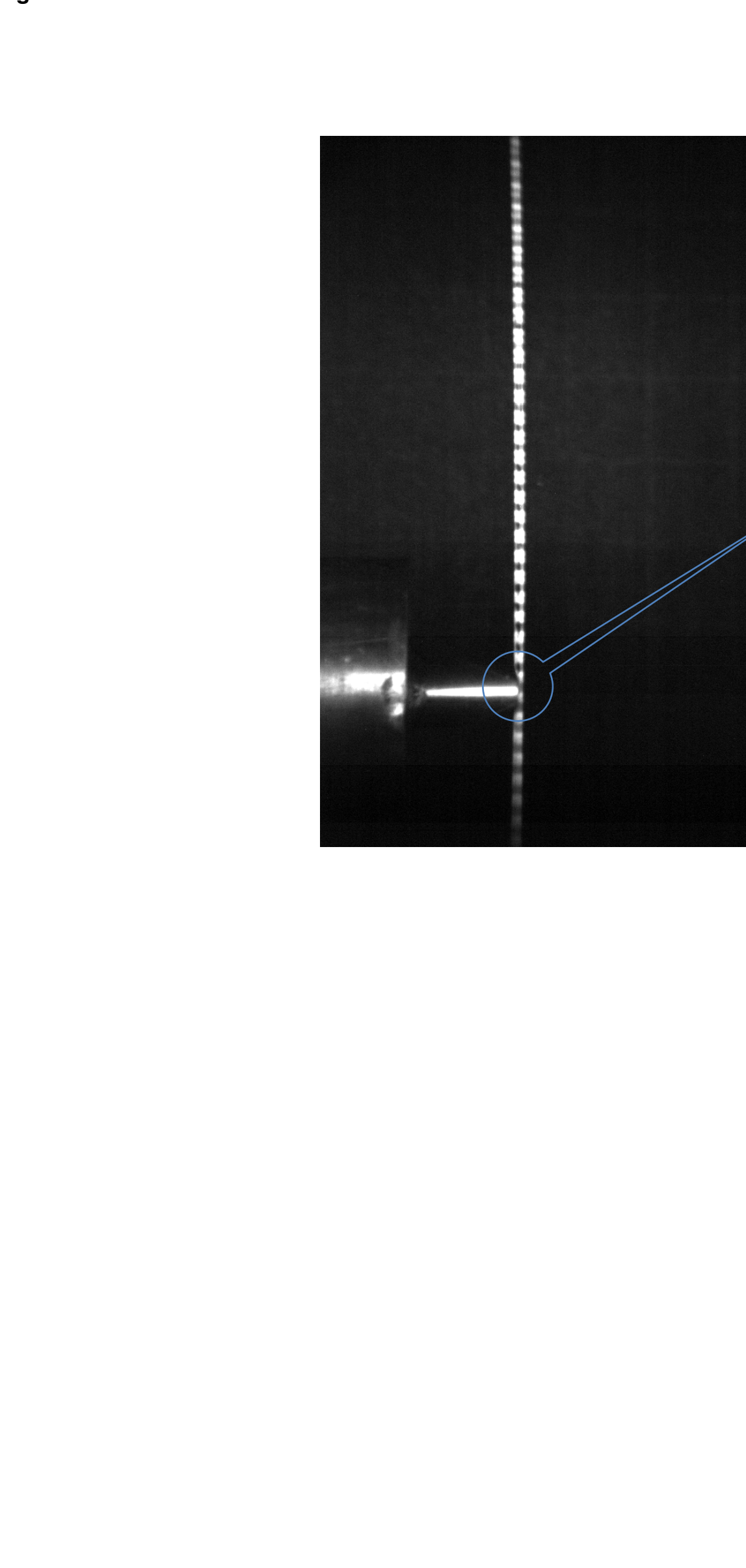

\section{Figures}




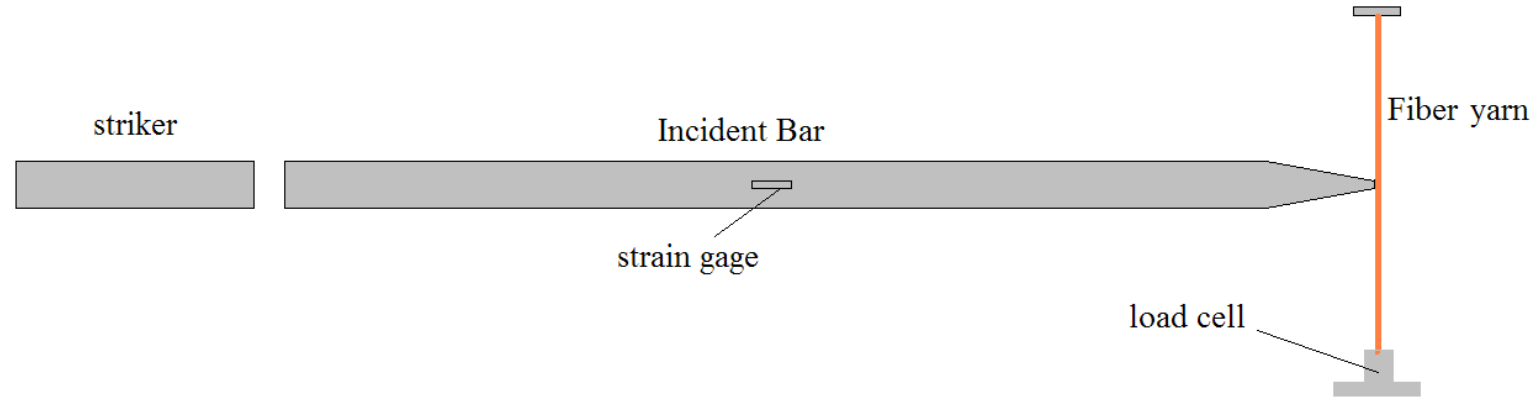

Figure 2 


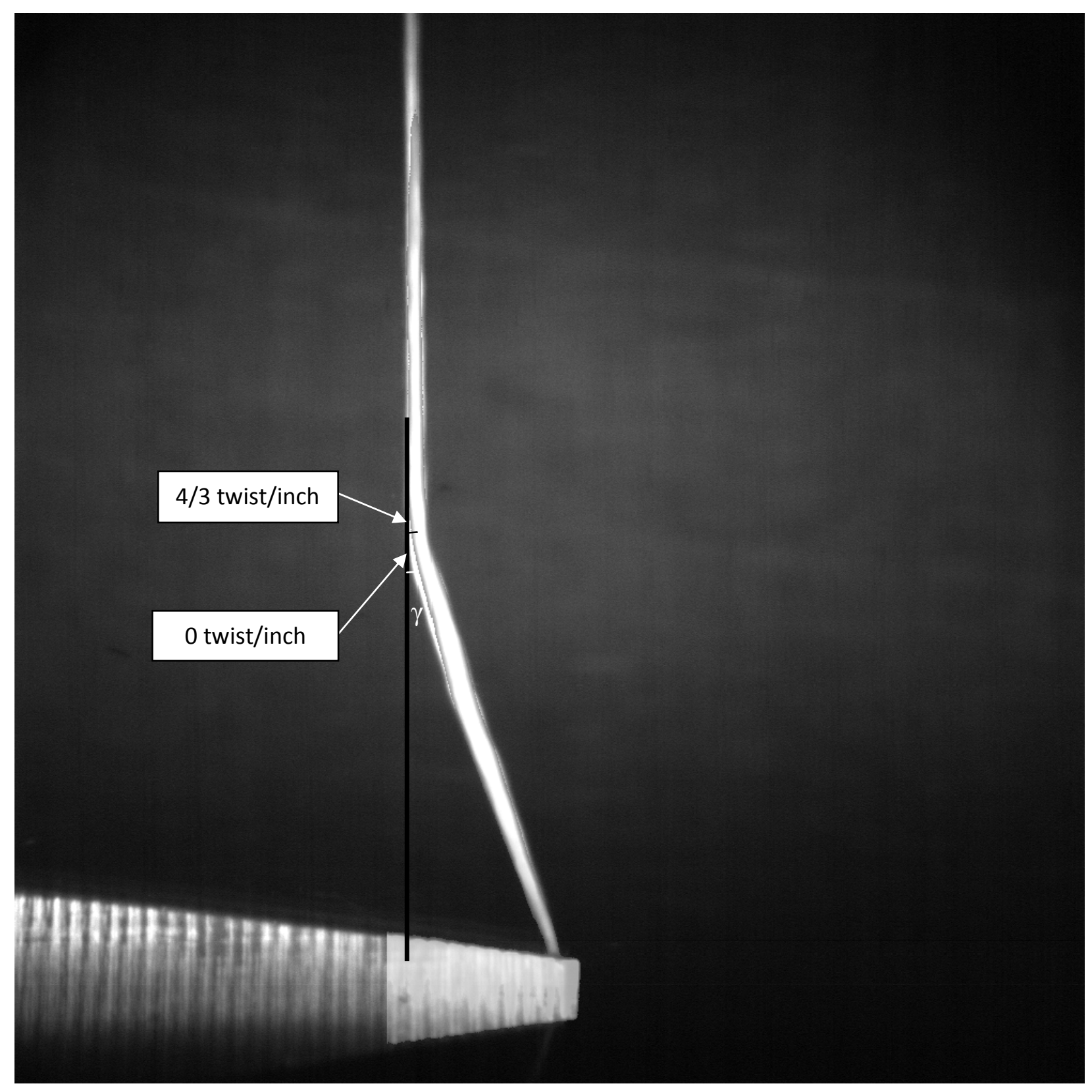

Figure 3 


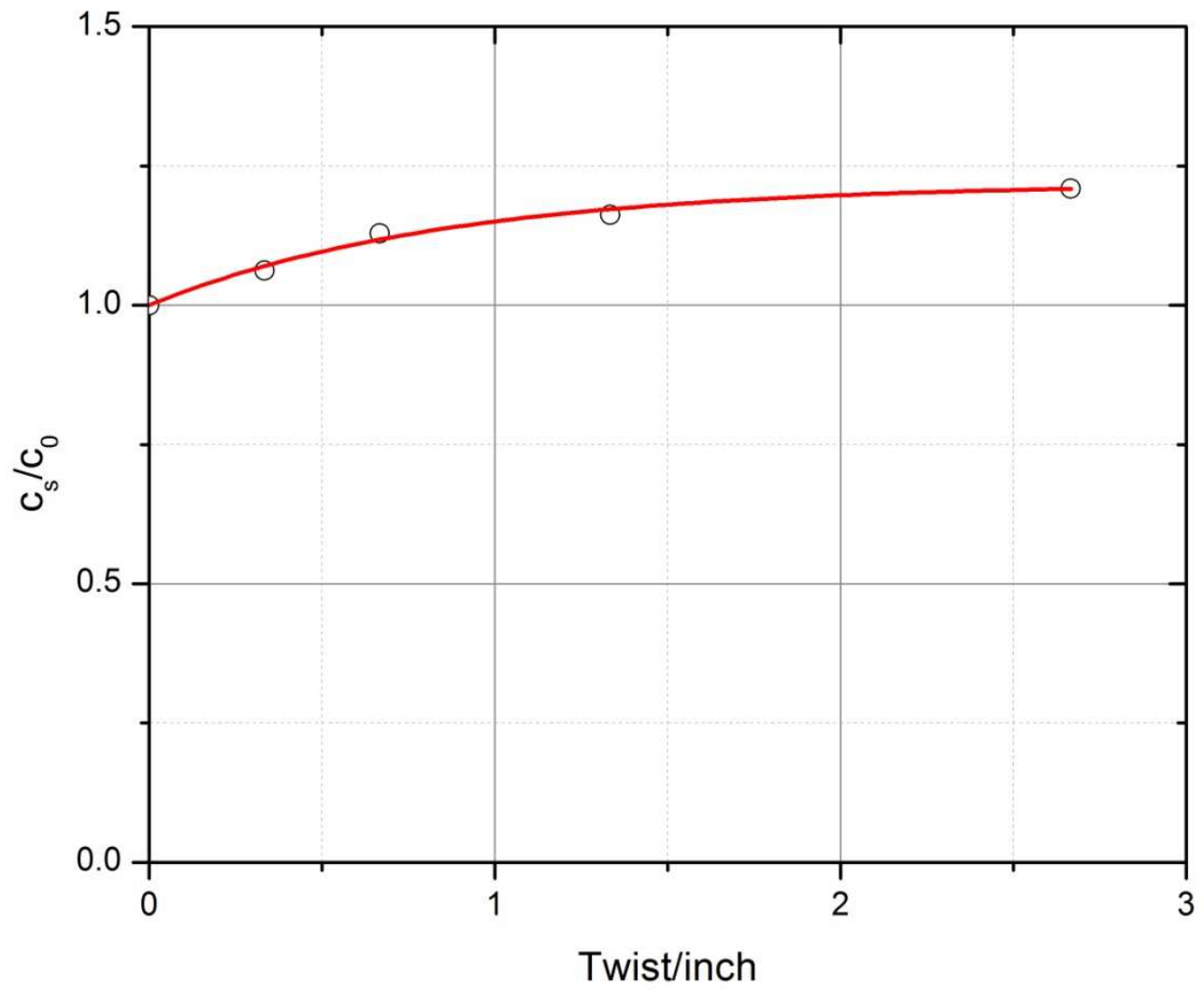

Figure 4 